\title{
Left-Sided Atrial Septal Pouch as a Risk Factor of Cryptogenic Stroke: A Systematic Review and Meta-Analysis
}

\author{
Mateusz K. Hołda Mateusz Koziej \\ HEART, Heart Embryology and Anatomy Research Team, Department of Anatomy, Jagiellonian University Medical \\ College, Cracow, Poland
}

\section{Keywords}

Interatrial septum · Left atrium · Ischemic stroke ·

Transesophageal echocardiography · Cardioembolic stroke - Embolic stroke of undetermined source

\section{Abstract}

Background: Despite some evidence of left-sided septal pouch (LSSP) involvement in the pathogenesis of cardioembolic stroke, the question of LSSP clinical significance still remains unsolved. In this study, we aimed to determine the association between the LSSP presence and cryptogenic stroke using meta-analytical approach. Methods: We performed a systematic review of electronic databases for studies that compared the presence of LSSP in subjects with cryptogenic stroke and non-stroke control. Data were extracted and pooled into a meta-analysis. Results: Seven studies (400 cryptogenic stroke patients and 1,456 nonstroke controls) were included in the meta-analysis. A total of 138 LSSPs were identified among the cryptogenic stroke patients, with a pooled prevalence of $29.8 \%(95 \%$ Cl $17.5-$ $43.7 \%$ ), and 268 LSSPs were identified in the non-stroke controls, with a pooled prevalence of $21.0 \%(95 \% \mathrm{Cl} 13.7-29.5 \%)$. After meta-analysis, the risk of cryptogenic stroke was high- er in patients with an LSSP than in patients without LSSP (OR $1.52 ; 95 \% \mathrm{Cl} 1.15-2.00 ; p<0.001)$. No significant heterogeneity was detected across the included studies $(p>0.05)$. Conclusion: Our meta-analysis demonstrated association between LSSP and cryptogenic stroke. In our univariate analysis, the risk of cryptogenic stroke is higher among patients with LSSP than in cases without the LSSP.

(C) 2018 S. Karger AG, Basel

\section{Introduction}

Cryptogenic stroke is defined as a symptomatic cerebral infarction for which no probable cause is identified after standard workup. Cryptogenic stroke is diagnosed based on the exclusion of known causes of stroke [1]. Ischemic stroke that remains cryptogenic after a standard clinical evaluation accounts for $20-30 \%$ of all ischemic strokes and is more prevalent among young adults [2].

Both of the authors take responsibility for all aspects of the reliability and freedom from bias of the data presented and their discussed interpretation. 
Numerous mechanisms for cryptogenic stroke have been proposed $[1,3]$, including thrombosis inside the left-sided septal pouch (LSSP) $[4,5]$.

The concept of the atrial septal pouch emerged in 2010 and was defined as a small, kangaroo pouch-like structure located on the human interatrial septum [4]. A septal pouch develops postnatally as a result of the partial fusion of the patent foramen ovale (PFO) channel. A diverticulum that arises by this mechanism can be located on either the left or right side of the interatrial septum, and there is typically no connection through the pouch between the right and left atrium [5].

The LSSP was immediately linked to cardioembolic events and proposed as an important thrombogenic site for stroke, similar to the left atrial appendage, which was confirmed by many case reports [4, 6-8]. In subsequent years, several original studies focused on the role of the LSSP in stroke patients. Unfortunately, based on the preliminary epidemiologic retrospective studies, the association between the presence of LSSP and increased risk of cryptogenic stroke is controversial [9-15]. Therefore, here we aimed to determine whether LSSP is associated with cryptogenic stroke using a meta-analytical approach.

\section{Methods}

\section{Search Strategy}

We adopted the Preferred Reporting Items for Systematic Reviews and Meta-Analyses guidelines for systematic reviews and meta-analyses [16]. We conducted a systematic literature search of the PubMed, EMBASE, and Scopus databases. The terms "septal pouch" OR "atrial septal pouch" were used to search the electronic databases. The search was not restricted by language or time. The last literature search flagged entries on May 2nd, 2018. We subsequently reviewed and evaluated the reference lists of all eligible articles to ensure the identification of all relevant studies.

\section{Eligibility Assessment and Data Extraction}

We required that the studies (1) evaluated the interatrial septum morphology in humans, (2) reported the prevalence of LSSP in subjects with cryptogenic stroke, and (3) compared those patients with non-stroke control patients. Articles that were eligible for inclusion in the meta-analysis were assessed by all authors. We initially excluded the following types of papers: case reports, editorials, conference abstracts, and duplicate reports of the same study. All authors independently extracted data from the included studies. The extracted data included the year, country, study design, samples size, and characteristics of each group, as well as the number of LSSPs identified in each group. Moreover, we also identified data regarding the distribution of other stroke risk factors, such as atrial fibrillation, coronary artery disease, congestive heart failure, dyslipidemia/hyperlipidemia, diabetes mellitus, arterial hypertension, and history of smoking among cryptogenic stroke

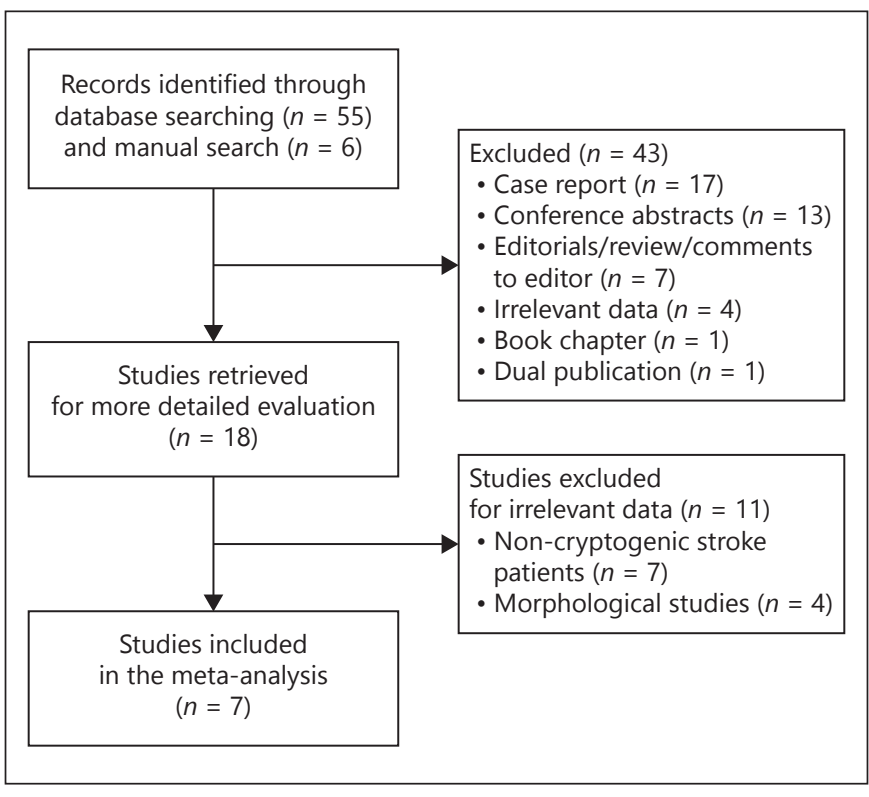

Fig. 1. Flow diagram demonstrating a selection of studies for metaanalysis. $n$, the number of records.

and non-stroke groups. In the event of data discrepancies, we contacted the authors of the original study, if possible, for further information. Any disagreements were addressed by obtaining a consensus.

\section{Statistical Analysis}

We performed a meta-analysis using the STATISTICA version 13.1 software (StatSoft Inc., Tulsa, OK, USA) with a Medical Bundle and MetaXL version 5.3 from EpiGear International Pty Ltd (Wilston, Queensland, Australia) to calculate the pooled prevalence of LSSPs. A $p$ value less than 0.05 was considered to be statistically significant. Evidence of publication bias was investigated using a funnel plot and analyzed using the Egger method. We assessed heterogeneity among the included studies using the Cochrane Handbook's Q test and $I^{2}$ statistics [17]. $p<0.05$ or $I^{2}>50 \%$ was considered to indicate significant heterogeneity. We applied the meta-analysis using a random-effects model. Qualitative variables were compared using the $\chi^{2}$ test of proportions for categorical variables. Sensitivity analysis was performed by omitting one study at a time to evaluate the influence of a single study on the overall estimate.

\section{Results}

An overview of the study identification and selection process is summarized in Figure 1. We initially identified 55 unique records. Six additional publications were identified after a manual search. Forty-three records were excluded for being case reports, conference abstracts, editorials, reviews, book chapters, or dual publi- 
cations or because they reported irrelevant data that were not related to septal pouches in the heart. Of the remaining 18 articles, we excluded 11 because they failed to report the prevalence of LSSPs in human subjects with ischemic stroke. Four studies were morphological studies $[4,5,18,19]$, and 7 studies were imaging studies without correlation with stroke subjects [20-26]. Next, we carefully assessed the studies for patients with cryptogenic stroke. We were able to identify the groups of patients with a history of cryptogenic stroke and the corresponding prevalence of LSSPs in 6 studies [9-13, 15]. There was no disagreement in the selection process. Due to unclear data, we contacted Sun et al. [14] to determine if the ischemic strokes identified in their study were cryptogenic. The authors confirmed that all of the ischemic strokes reported in their study were cryptogenic strokes of unknown etiology [14]. In summary, 7 studies that met our initial criteria were included in the metaanalysis. Funnel plot analysis does not reveal a significant publication bias influencing the data (Egger's test: $p=0.98)$.

The characteristics and results of the included studies are presented in Table 1. Six studies were TEE studies [9-11, 13-15], and 1 study was a computed tomography study [12]. Distribution of other stroke risk factors among groups in studies included into a meta-analysis is presented in online supplementary Table 1 (for all online suppl. material, see www.karger. com/doi/10.1159/000495573). All included studies comprised adult patients, but there was a significant difference in patient age between studies (Table 1). Cryptogenic stroke, which was briefly defined as an ischemic stroke with no definite source despite a thorough diagnostic evaluation, was recognized in 400 patients. A total of 1,456 non-stroke patients served as controls. A total of 138 LSSPs were identified among the cryptogenic stroke patients, with a pooled prevalence of $29.8 \%$ (95\% CI 17.5-43.7\%; random-effects model; Fig. 2a), and 268 LSSPs were identified in the non-stroke controls, with a pooled prevalence of $21.0 \%$ (95\% CI $13.7-$ 29.5\%; random-effects model; Fig. 2b). After meta-analysis, the risk of cryptogenic stroke was higher in patients with an LSSP than in patients without an LSSP (OR 1.52; 95\% CI 1.15-2.00; $p<0.001$; random-effect model; Fig. 3). No evidence of heterogeneity was found in the analysis $\left(\mathrm{I}^{2}=0.92 \% ; p=0.42\right)$. Results of the sensitivity analysis are presented in online supplementary Figure 1. We have observed that only exclusion of the Hołda et al. [15] study influenced the pooled estimates which remained on the verge of statistical significance.

LSSP and Cryptogenic Stroke

\section{Discussion}

Almost all morphological features of LSSP predispose to local blood stasis and thrombosis inside the pouch. The LSSP volume makes up only about $15 \%$ of the left atrial appendage volume. However, pouch orientation (the LSSP apex is always directed downward) might be more predisposed to thrombus formation [5]. Moreover, the LSSP was found to be associated with a 2 -fold increased risk of atrial fibrillation [20]. Combination of these 2 factors might be a reasonable explanation for why the LSSP is associated with increased risk of cryptogenic stroke occurrence.

Our systematic review and meta-analysis show that cryptogenic stroke occurred 1.5-times more frequently in patients with an LSSP. A previous meta-analysis performed by Strachinaru et al. [27] focused on the presence of LSSP in patients with ischemic stroke as a whole group, as well as in a subset of patients with cryptogenic stroke. In their random-effects meta-analysis, Strachinaru et al. [27] detected no difference in LSSP prevalence between non-stroke controls and patients with ischemic stroke (HR 1.20; 95\% Cl 0.96-1.53; $p=0.14$ ). This was not surprising, as many other factors might be responsible for the occurrence of stroke, and the results of that metaanalysis were not adjusted for other stroke risk factors. Cryptogenic stroke was shown to appear more frequently in patients with LSSP (HR 1.53; 95\% Cl 1.07-2.24; $p=$ 0.02 ), but this was driven by only a single underpowered, small study performed by Wong et al. [11]. Therefore, consensus on the possible association between LSSP and cryptogenic stroke was not reached. The meta-analysis performed by Strachinaru et al. [27] did not include 3 studies that comprised current meta-analyses $[12,14$, 15].

Sensitivity analysis showed that our meta-analysis is well balanced, and exclusion of only one study, which was the study conducted by Hołda et al. [15], that also has the highest weight in our meta-analysis influenced the pooled estimates, shifting them to the lower border and on the verge of statistical significance $(p=0.051)$. Exclusion of studies performed by Sun et al. [14] or Wong et al. [11] also shifted the results to lower values, but the overall model was still statistically significant $(p<0.05)$. Exclusion of 3 studies such as by Tugcu et al. [9], Wayangankar et al. [10], and Strachinaru et al. [13] made the results of the meta-analysis more significant.

The limitations of this meta-analysis are caused by the disadvantages of each study included. Although the definition of cryptogenic stroke was similar in all in-

Cerebrovasc Dis 2018;46:223-229 


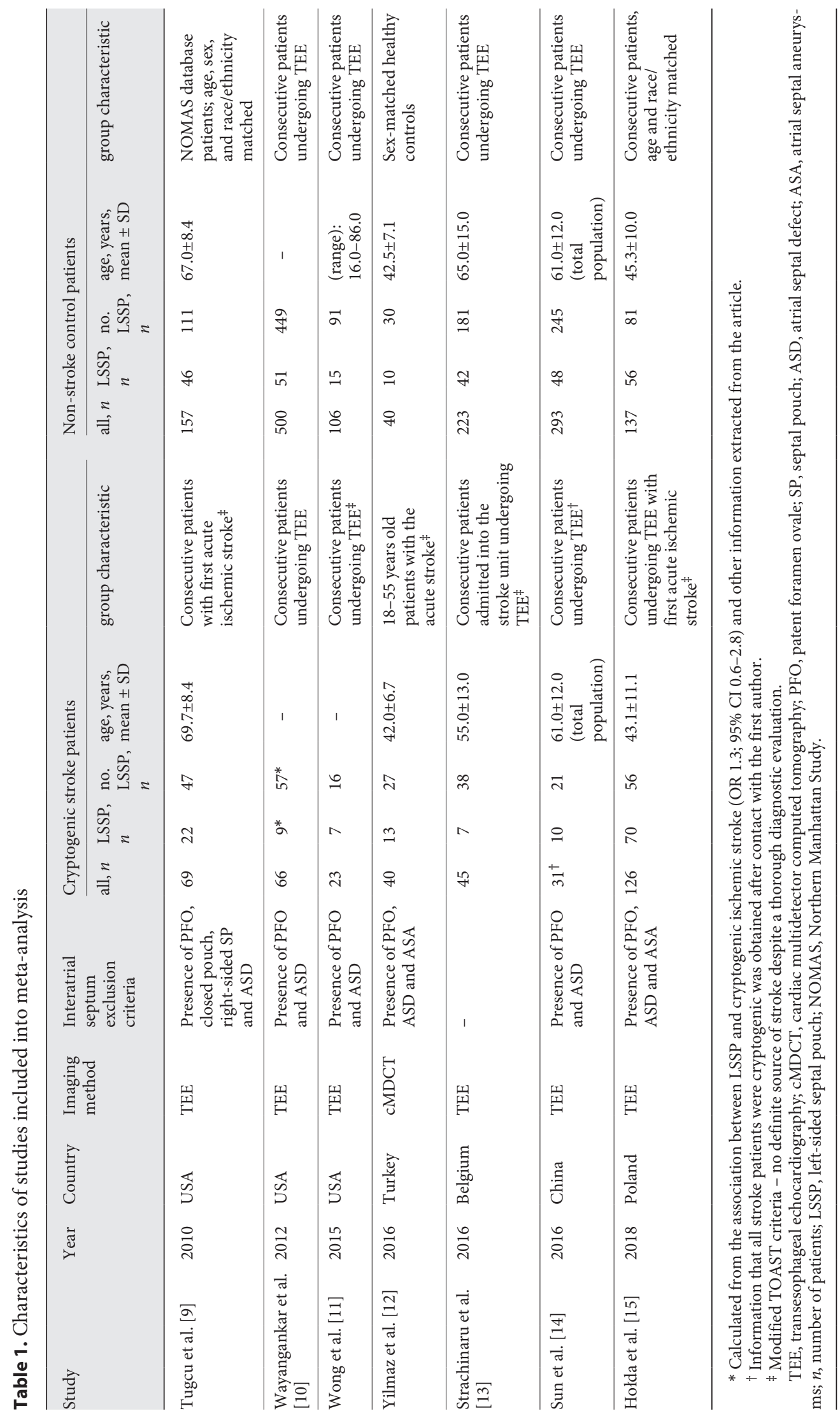




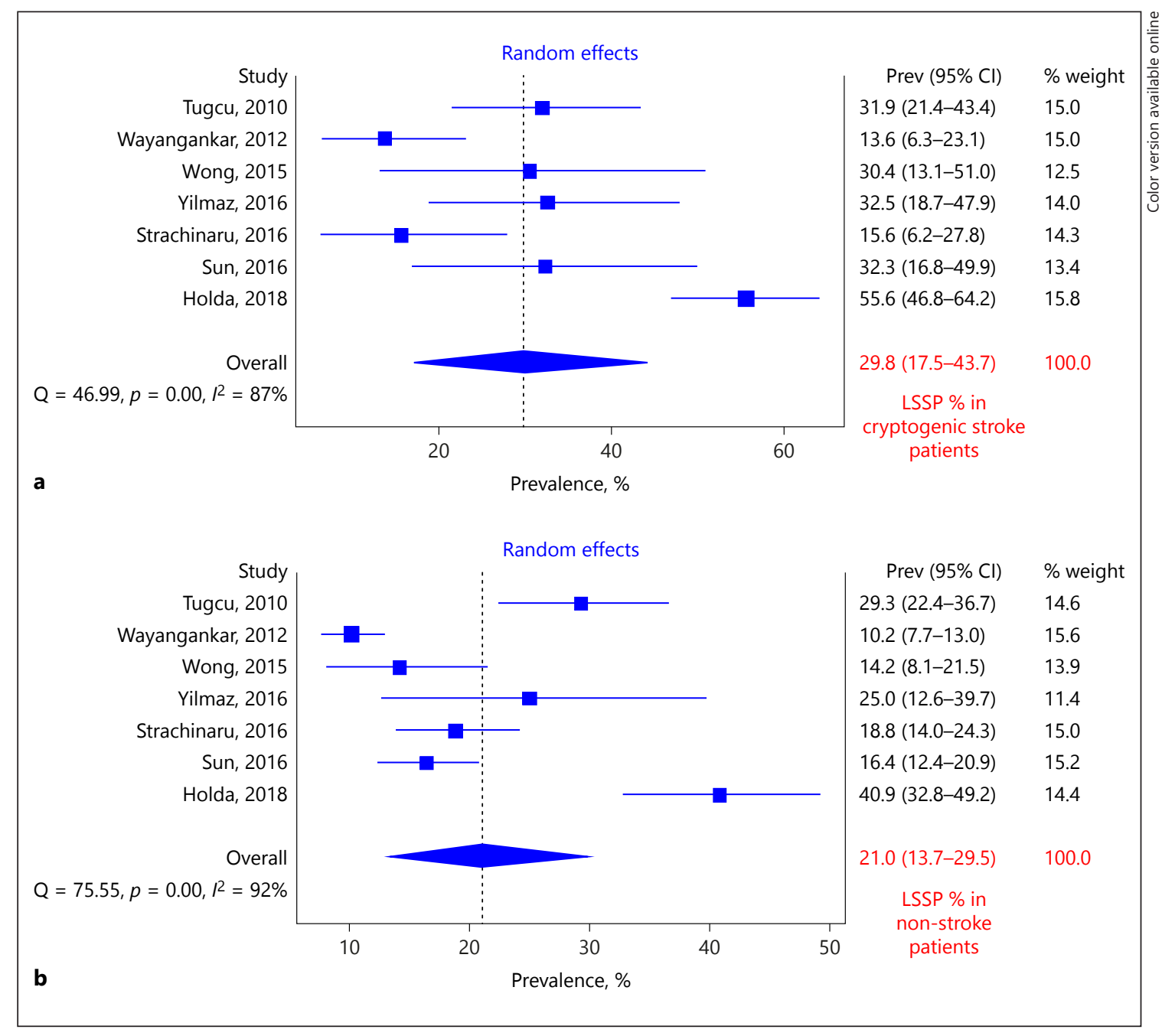

Fig. 2. Forest plots of the prevalence of the LSSP in (a) cryptogenic stroke and (b) non-stroke control patients. LSSP, left-sided septal pouch.

cluded studies (modified TOAST criteria), some discrepancies in the study group and control selection processes might affect the final results. One of the studies performed by Strachinaru et al. [13] did not exclude patients with the presence of a PFO and atrial septal defects. Such patients were excluded in the remaining studies because these structures have previously been associated with cryptogenic stroke (Table 1) [28]. Also, a significant number of patients were excluded from the included studies at baseline because the interatrial septum was not well-visualized or because saline contrast injection was not performed. All of the studies were retrospective cross-sectional studies; therefore, we were unable to determine whether having an LSSP increases cryptogenic stroke risk longitudinally. Additionally, the imaging method (transesophageal echocardiography and cardiac computed tomography) may have negatively affected the results due to inadequate visualization of the LSSP, which would have understated the prevalence of this feature in both groups. A study that compares different imaging methods for the LSSP is required to define the best imaging technique and projection [29]. Moreover, many cryptogenic stroke patients do not routinely undergo transesophageal echocardiography; as a result, all studies are subject to patients selection bias.

The main factor that might affect the results of the meta-analysis is the age of the patients. The remodeling of 
Fig. 3. Forest plot representation of the results from the significant studies comparing cryptogenic stroke with controls. Studies are displayed on the vertical and are marked with a square of proportional size to the study's calculated weight. The overall effect is lowermost and marked with a rhombus.

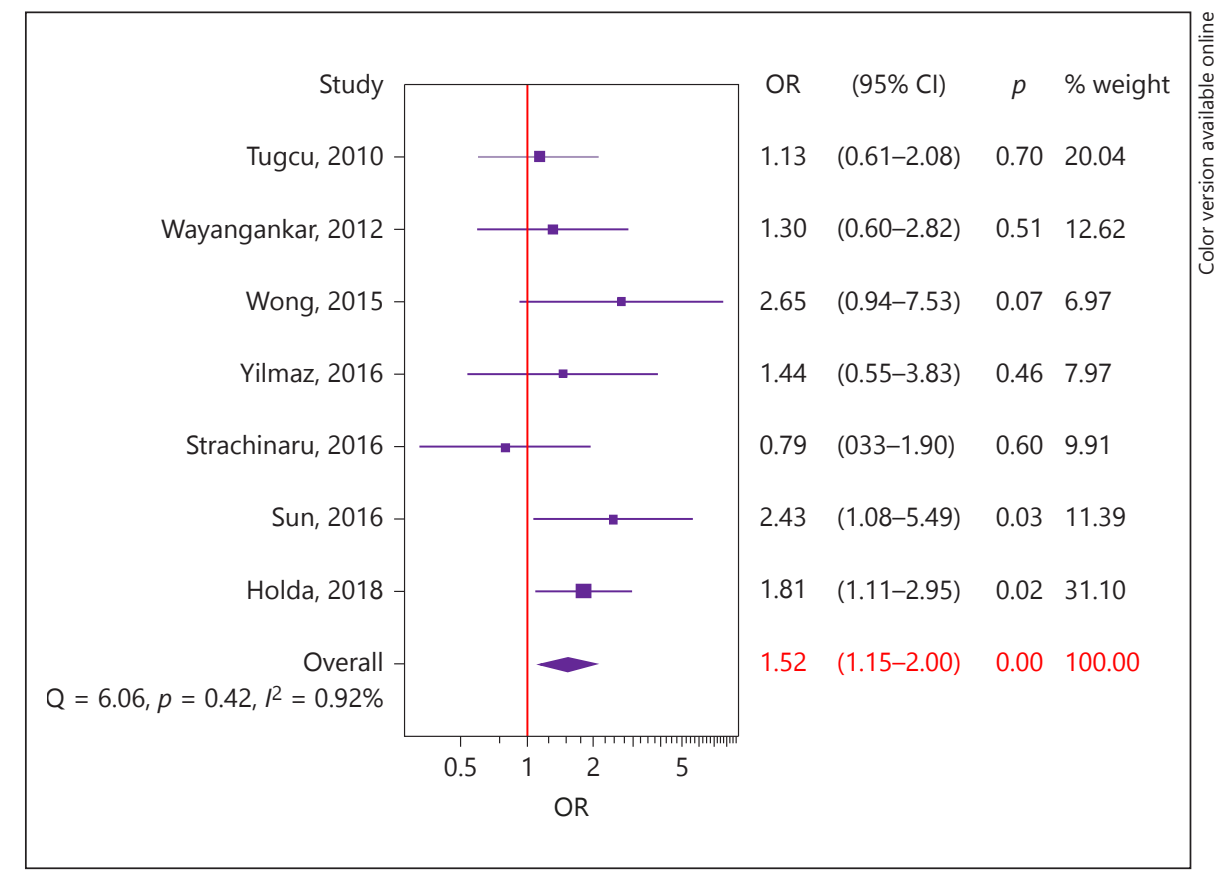

the interatrial septum is a continuous process in which the PFO channel evolves into a smooth septum or septal pouch. The prevalence of the LSSP is the highest among young adults and significantly lower in older patients, in which more cases with a smooth septum could be noticed $[5,9]$. The mean age of the subjects included in each study group differed significantly between the non-stroke and cryptogenic stroke groups and between studies. Among the 7 studies included in the meta-analysis, only the studies performed by Yilmaz et al. [12], Wong et al. [11], and Hołda et al. [15] included young adult populations (Table 1). The cryptogenic stroke and non-stroke populations in the study performed by Tugcu et al. [9] were the oldest among all of the included studies, which may have influenced the results of this study and the original study's conclusion that there is no significant association between LASP and cryptogenic stroke. In the study by Strachinaru et al. [13], the cryptogenic stroke group was significantly younger than the non-stroke controls (55.0 \pm 13.0 vs. $65.0 \pm 15.0$-years-old; $p<0.0001$ ), which may also have affected the results of this study, where in older patients the smooth septum might prevail over LSSP, and in younger patients the PFO might prevail over LSSP. As those studies by Tugcu et al. [9] and Strachinaru et al. [13] have a large weight (30\%), the results of the meta-analysis might be affected, and the real association between LSSP and cryptogenic stroke in young adults may differ. However, our sensitivity analysis did not show that the exclu- sion of any of this study makes analysis insignificant (online suppl. Fig. 1). No information about patient age was provided by Wayangankar et al. [10]. Since the ethnic differences in the LSSP prevalence are not recognized, they should be accounted for in further studies. Finally, the associations between LSSP and other types of cerebrovascular and systemic thromboembolic events should also be investigated.

\section{Conclusion}

In conclusion, our meta-analysis demonstrated an association between LSSP and cryptogenic stroke. In our univariate analysis, the risk of cryptogenic stroke was higher among patients with LSSP than in cases without the LSSP.

\section{Acknowledgments}

Dr. Mateusz K. Hołda was supported by the Foundation for Polish Science.

\section{Disclosure Statement}

The authors declare no conflicts of interest in relation to the current work. 


\section{References}

1 Nouh A, Hussain M, Mehta T, Yaghi S: Embolic strokes of unknown source and cryptogenic stroke: implications in clinical practice. Front Neurol 2016;7:37.

2 Hart RG, Diener HC, Coutts SB, Easton JD, Granger CB, O’Donnell MJ, et al: Embolic strokes of undetermined source: the case for a new clinical construct. Lancet Neurol 2014; 13:429-438.

3 Saver JL: Cryptogenic stroke. N Engl J Med 2016;375:e26.

4 Krishnan SC, Salazar M: Septal pouch in the left atrium: a new anatomical entity with potential for embolic complications. JACC Cardiovasc Interv 2010;3:98-104.

5 Hołda MK, Koziej M, Hołda J, Piątek K, Tyrak K, Chołopiak W, et al: Atrial septal pouch morphological features and clinical considerations. Int J Cardiol 2016;220:337-342.

6 Strachinaru M, Morissens M, Latifyan S, Costescu I: Left atrial septal pouch thrombus assessed on three-dimensional transoesophageal echocardiography. Eur Hear J Cardiovasc Imaging 2012;13:967.

7 Wong JM, Lombardo D, Handwerker J, Fisher M: Cryptogenic stroke and the left atrial septal pouch: a case report. J Stroke Cerebrovasc Dis 2014;23:564-575.

8 Aggarwal S, Kalavakunta J, Gupta V: Left atrial septal pouch thrombus: a common pathology in an uncommon location. Int J Cardiol 2016;212:369-370.

9 Tugcu A, Okajima K, Jin Z, Rundek T, Homma S, Sacco RL, et al: Septal pouch in the left atrium and risk of ischemic stroke. JACC Cardiovasc Imaging 2010;3:1276-1283.

10 Wayangankar SA, Patel JH, Patel B, Stavrakis S, Sivaram CA: Clinical and echocardiographic variables associated with LA septal pouch. JACC Cardiovasc Imaging 2013;6:833-835.

11 Wong JM, Lombardo DM, Barseghian A Dhoot J, Hundal HS, Salcedo J, et al: Left atrial septal pouch in cryptogenic stroke. Front Neurol 2015;6:57.
12 Yilmaz M, Vural MG, Karcaaltincaba M, Yoldas TK, Yilmaz MS, Kavalci C: Left-sided atrial septal pouch and risk of cryptogenic stroke. Acta Medica Mediterr 2016;32:785789.

13 Strachinaru M, Catez E, Jousten I, Pavel O, Janssen C, Morissens M, et al: The left atrial septal pouch as a possible risk factor for stroke. Echocardiography 2016;33:1016-1023.

14 Sun JP, Meng F, Yang XS, Lee AP, Chen M, Zhang B, et al: Prevalence of atrial septal pouch and risk of ischemic stroke. Int J Cardiol 2016;214:37-40.

15 Hołda M, Krawczyk-Ożóg A, Koziej M, Sorysz D, Hołda J, Dudek D, et al: Left-sided atrial septal pouch is a risk factor for cryptogenic stroke. J Am Soc Echocardiogr 2018;31: 771-776.

16 Liberati A, Altman DG, Tetzlaff J, Mulrow C, Gøtzsche PC, Ioannidis JP, et al: The PRISMA statement for reporting systematic reviews and meta-analyses of studies that evaluate health care interventions: explanation and elaboration. J Clin Epidemiol 2009;62:e1-e34.

17 Higgins JP, Thompson SG, Deeks JJ, Altman DG: Measuring inconsistency in meta-analyses. BMJ 2003;327:557-560.

18 Klimek-Piotrowska W, Hołda MK, Koziej M, Piątek K, Hołda J: Anatomy of the true interatrial septum for transseptal access to the left atrium. Ann Anat 2016;205:60-64.

19 Hołda MK, Hołda J, Koziej M, Piątek K, Klimek-Piotrowska W: Porcine heart interatrial septum anatomy. Ann Anat 2018;217: 24-28.

20 Hołda MK, Koziej M, Wszołek K, Pawlik W, Krawczyk-Ożóg A, Sorysz D, et al: Left atrial accessory appendages, diverticula, and leftsided septal pouch in multi-slice computed tomography. Association with atrial fibrillation and cerebrovascular accidents. Int J Cardiol 2017;244:163-168.

21 Elsayed M, Hsiung MC, Meggo-Quiroz LD, Elguindy M, Uygur B, Tandon R, et al: Incre- mental value of live/real time three-dimensional over two-dimensional transesophageal echocardiography in the assessment of atrial septal pouch. Echocardiography 2015;32: 1858-1867.

22 Vehian A, Choi B, Rekhi S, Young H, Dusaj $\mathrm{R}$, Zeman R: Clinical significance of left atrial anatomic abnormalities identified by cardiac computed tomography. Adv Comput Tomogr 2015;4:1-8.

23 Terpenning S, Ketai L, Rissing S, Teague S: Correlation of left atrial septal pouch with the prevalence of Patent foramen ovale: a retrospective review. Cardiol Angiol An Int J 2015; 3:122-129.

24 Hołda MK, Krawczyk-Ożóg A, Koziej M, Sorysz D, Hołda J, Dudek D, Klimek-Piotrowska W: Mid-esophageal bicaval versus short-axis view of interatrial septum in two-dimensional transesophageal echocardiography for diagnosis and measurement of atrial septal pouches. Echocardiography 2018;35:827833

25 Hołda MK, Krawczyk-Ożóg A, Koziej M, Pawlik W, Sorysz D, Wszołek K, et al: Cardiac computed tomography compared with twodimensional transesophageal echocardiography for the detection and assessment of atrial septal pouches. Int J Cardiovasc Imaging 2018;34:1305-1313.

26 Kabirdas D, Nekkanti R: Webbed left atrial septal pouch-A new anatomical variant. Echocardiography 2018;35:889-892.

27 Strachinaru M, Castro-Rodriguez J, Verbeet T, Gazagnes MD: The left atrial septal pouch as a risk factor for stroke: a systematic review. Arch Cardiovasc Dis 2017;110:250-258.

28 Handke M, Harloff A, Olschewski M, Hetzel A, Geibel A: Patent foramen ovale and cryptogenic stroke in older patients. N Engl J Med 2007;357:2262-2268.

29 Wong JM, Fisher M: The potential role of the left atrial septal pouch in cryptogenic stroke. Expert Rev Cardiovasc Ther 2016;14:1-3. 